\title{
Majorana fermions in $s$-wave noncentrosymmetric superconductor with Dresselhaus (110) spin-orbit coupling
}

\author{
Jiabin You ${ }^{1,}$. C. H. Oh ${ }^{1, \text { † }}$ and Vlatko Vedral ${ }^{1,2}$ 用 \\ ${ }^{1}$ Centre for Quantum Technologies and Department of Physics, \\ National University of Singapore, 117543, Singapore \\ ${ }^{2}$ Department of Physics, University of Oxford, Clarendon Laboratory, Oxford, OX1 3PU, United Kingdom
}

(Dated: June 22, 2021)

\begin{abstract}
The asymmetric spin-orbit interactions play a crucial role in realizing topological phases in noncentrosymmetric superconductor (NCS). We investigate the edge states and the vortex core states in the $s$-wave NCS with Dresselhaus (110) spin-orbit coupling by both numerical and analytical methods. In particular, we demonstrate that there exists a novel semimetal phase characterized by the flat Andreev bound states in the phase diagram of the $s$-wave Dresselhaus NCS which supports the emergence of Majorana fermions. The flat dispersion implies a peak in the density of states which has a clear experimental signature in the tunneling conductance measurements and the Majorana fermions proposed here should be experimentally detectable.
\end{abstract}

PACS numbers: 03.65.Vf, 05.30.Rt, 74.25.Uv, 73.21.-b, 74.78.-w

\section{INTRODUCTION}

Topological phase of condensed matter systems is a quantum many-body state with nontrivial momentum or real space topology in the Hilbert spaces 10 . Recent newly discovered topological superconductor (TSC) has spawned considerable interests since this kind of topological phase supports the emergence of Majorana fermion $(\mathrm{MF}) \sqrt{9}$ which is a promising candidate for the faulttolerant topological quantum computation (TQC) 14 . There are several proposals for hosting MFs in TSC, for example, chiral $p$-wave superconductor ${ }^{9}$, $\mathrm{Cu}$-doped topological insulator $\mathrm{Bi}_{2} \mathrm{Se}_{3} \frac{15}{15}$, superconducting proximity devices $12[16,19]$ and noncentrosymmetric superconductor (NCS) 10 . The signatures of MFs have also been reported in the superconducting InSb nanowire ${ }^{20}, \mathrm{Cu}_{x} \mathrm{Bi}_{2} \mathrm{Se}_{3} 21$ and topological insulator Josephson junction 22 . To obtain a readily manipulated Majorana platform for TQC, more experimental confirmations and theoretical proposals are therefore highly desirable.

In this paper, we study the topological phase and Majorana fermion at the edge and in the vortex core of the $s$-wave Dresselhaus (110) spin-orbit (SO) coupled NCS. It is found that the asymmetric SO interaction plays a crucial role in realizing topological phases in the NCS. Although the Rashba SO coupled NCS has been previously investigated ${ }^{10 \mid 23}$, the Dresselhaus (110) SO coupled NCS is relatively less discussed theoretically 18 . Interestingly, we find that there is a novel semimetal phase in the Dresselhaus NCS, where the energy gap closes in the whole region and different kinds of flat Andreev bound states (ABSs) emerge. We demonstrate that these flat ABSs support the emergence of MFs analytically and numerically. It is known that the Chern number is not a welldefined topological invariant in the gapless region, however, we find that the topologically different semimetal phases in this gapless region can still be distinguished by the Pfaffian invariant of the particle-hole symmetric Hamiltonian.
Several authors have proposed the flat ABSs in the NCS $\mathrm{Li}_{2} \mathrm{Pd}_{x} \mathrm{Pt}_{3-x} \mathrm{~B}$ with high order SO couplings $24 \mid 25$, $d_{x y}$-wave superconductor, $p_{x}$-wave superconductor and $d_{x y}+p$-wave superconductor ${ }^{26}$. Instead, our proposal for hosting the flat ABSs is an $s$-wave Dresselhaus (110) SO coupled NCS in an in-plane magnetic field which is more flexible than the previous proposals where one needs to apply a magnetic field in the $y$ direction to

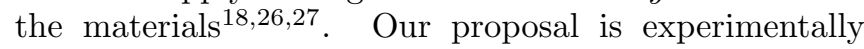
more feasible. The flat dispersion implies a peak in the density of states (DOS) which is clearly visible and has an experimental signature in the tunneling conductance measurements 28 . The zero-bias conductance peak has been observed in recent experiments on the $\mathrm{InSb}$ nanowire ${ }^{20}$ and $\mathrm{Cu}_{x} \mathrm{Bi}_{2} \mathrm{Se}_{3} 21$ and argued to be due to the flat ABS. Thus if the Majorana fermion exists in the Dresselhaus NCS, the flat ABS and the zero-bias conductance peak in the DOS predicted here should be detectable.

The paper is organized as follows. The model for $s$ wave NCS with Dresselhaus (110) SO coupling is given in Sec. II]. The phase diagrams and topological invariants of this model are discussed in Sec. III. The numerical and analytical solutions to the Majorana fermions at the edge of the system are demonstrated in Sec. IV. The Majorana fermions in the vortex core of the system are numerically shown in Sec. V] Finally, we give a brief summary in Sec. VI.

\section{MODEL}

We begin with modeling the Hamiltonian in a square lattice for the two dimensional $s$-wave NCS with Dresselhaus (110) SO interaction in an in-plane magnetic field, 
which is given by $H=H_{\text {kin }}+H_{\mathrm{Z}}+H_{\mathrm{D}}^{110}+H_{s}$ :

$$
\begin{aligned}
H_{\text {kin }} & =-t \sum_{i s} \sum_{\hat{\nu}=\hat{x}, \hat{y}}\left(c_{i+\hat{\nu} s}^{\dagger} c_{i s}+c_{i-\hat{\nu} s}^{\dagger} c_{i s}\right)-\mu \sum_{i s} c_{i s}^{\dagger} c_{i s}, \\
H_{\mathrm{Z}} & =\sum_{i s s^{\prime}}(\mathbf{V} \cdot \sigma)_{s s^{\prime}} c_{i s}^{\dagger} c_{i s^{\prime}}, \\
H_{\mathrm{D}}^{110} & =-i \frac{\beta}{2} \sum_{i s s^{\prime}}\left(\sigma_{z}\right)_{s s^{\prime}}\left(c_{i-\hat{x} s}^{\dagger} c_{i s^{\prime}}-c_{i+\hat{x} s}^{\dagger} c_{i s^{\prime}}\right), \\
H_{s} & =\sum_{i}\left[\Delta_{s} c_{i \uparrow}^{\dagger} c_{i \downarrow}^{\dagger}+\text { H.c. }\right],
\end{aligned}
$$

where $c_{i s}^{\dagger}\left(c_{i s}\right)$ denotes the creation (annihilation) operator of the electron with spin $s=(\uparrow, \downarrow)$ at site $i=\left(i_{x}, i_{y}\right)$. $H_{\text {kin }}$ is the hopping term with hopping amplitude $t$ and chemical potential $\mu . H_{\mathrm{Z}}$ is the Zeeman field induced by the in-plane magnetic field with components $\mathbf{V}=$ $\left(V_{x}, V_{y}, 0\right)=\frac{g \mu_{B}}{2}\left(B_{x}, B_{y}, 0\right) . \quad H_{\mathrm{D}}^{110}$ is the Dresselhaus (110) SO coupling and $H_{s}$ is the $s$-wave superconducting term with gap function $\Delta_{s}$. We assume $t>0$ throughout this paper. In the momentum space, the Hamiltonian is $H=\frac{1}{2} \sum_{\mathbf{k}} \psi_{\mathbf{k}}^{\dagger} \mathcal{H}(\mathbf{k}) \psi_{\mathbf{k}}$ with $\psi_{\mathbf{k}}^{\dagger}=\left(c_{\mathbf{k} \uparrow}^{\dagger}, c_{\mathbf{k} \downarrow}^{\dagger}, c_{-\mathbf{k} \uparrow}, c_{-\mathbf{k} \downarrow}\right)$, where $c_{\mathbf{k} s}^{\dagger}=(1 / \sqrt{N}) \sum_{\mathbf{l}} e^{i \mathbf{k} \cdot{ }_{1}} c_{1 s}^{\dagger}, \mathbf{k}$ is the wave vector in the first Brillouin zone and the Bogoliubov-de Gennes (BdG) Hamiltonian is

$$
\mathcal{H}(\mathbf{k})=\xi_{\mathbf{k}} \sigma_{z}+\beta \sin k_{x} \tau_{z}+V_{x} \sigma_{z} \tau_{x}+V_{y} \tau_{y}-\Delta_{s} \sigma_{y} \tau_{y}
$$

where $\xi_{\mathbf{k}}=-2 t\left(\cos k_{x}+\cos k_{y}\right)-\mu, \sigma$ and $\tau$ are the Pauli matrices operating on the particle-hole space and spin space, respectively. The nontrivial topological order in the Dresselhaus NCS is characterized by the existence of gapless edge state and Majorana fermion. Below we shall demonstrate these features in the Hamiltonian Eq. (1).

\section{PHASE DIAGRAMS AND TOPOLOGICAL INVARIANTS}

For comparison, we first briefly summarize the known results of the $s$-wave Rashba NCS, in which the Dresselhaus (110) SO coupling $H_{\mathrm{D}}^{110}$ in the Hamiltonian Eq. (1) is replaced by the Rashba SO coupling $H_{\mathrm{R}}=$ $-\frac{\alpha}{2} \sum_{i}\left[\left(c_{i-\hat{x} \downarrow}^{\dagger} c_{i \uparrow}-c_{i+\hat{x} \downarrow}^{\dagger} c_{i \uparrow}\right)+i\left(c_{i-\hat{y} \downarrow}^{\dagger} c_{i \uparrow}-c_{i+\hat{y} \downarrow}^{\dagger} c_{i \uparrow}\right)+\right.$ H.c.] and the in-plane magnetic field is replaced by a perpendicular magnetic field 10 . As usual, we can use the Chern number to characterize the nontrivial momentum space topology of the Rashba NCS. The Chern number defined for the fully gapped Hamiltonian is $\mathcal{C}=\frac{1}{2 \pi} \int_{T^{2}} d k_{x} d k_{y} \mathcal{F}(\mathbf{k})$, where $\mathcal{F}(\mathbf{k})=$ $\epsilon^{i j} \partial_{k_{i}} A_{j}(\mathbf{k})$ is the strength of the gauge field $A_{i}(\mathbf{k})=$ $i \sum_{o c c .}\left\langle\psi_{n}(\mathbf{k}) \mid \partial_{k_{i}} \psi_{n}(\mathbf{k})\right\rangle$, where $\psi_{n}(\mathbf{k})$ is the eigenstates of the Hamiltonian. The integral is carried out in the first Brillouin zone and the summation is carried out for the occupied states. As long as the topological quantum transition does not happen, the Chern number remains unchanged. Since the topological quantum transition happens when the energy gap closes, the phase boundary can be depicted by studying the gap-closing condition of the Hamiltonian. In the phase diagram of the Rashba NCS as shown in the Fig. (11), we find that the gap closes in some lines and the Chern number is attached to each region of the phase diagram.

However, in the present case, we shall show that the phase diagram of the Dresselhaus NCS has a gapless region that makes the Chern number ill-defined. To see this, we diagonalize the $\mathrm{BdG}$ Hamiltonian Eq. (2) in the periodic boundary conditions of the $x$ and $y$ directions, then the energy spectrum is $E(\mathbf{k})=$ $\pm \sqrt{\xi_{\mathbf{k}}^{2}+\mathcal{L}_{\mathbf{k}}^{2}+V^{2}+\Delta_{s}^{2} \pm 2 \sqrt{\xi_{\mathbf{k}}^{2} \mathcal{L}_{\mathbf{k}}^{2}+V^{2}\left(\xi_{\mathbf{k}}^{2}+\Delta_{s}^{2}\right)}}$,

where $V=\sqrt{V_{x}^{2}+V_{y}^{2}}$ and $\mathcal{L}_{\mathbf{k}}=\beta \sin k_{x}$. Therefore, we can find that the energy gap closes at $\xi_{\mathbf{k}}^{2}+\mathcal{L}_{\mathbf{k}}^{2}+V^{2}+\Delta_{s}^{2}=2 \sqrt{\xi_{\mathbf{k}}^{2} \mathcal{L}_{\mathbf{k}}^{2}+V^{2}\left(\xi_{\mathbf{k}}^{2}+\Delta_{s}^{2}\right)}$ which leads to the following gap-closing conditions: $\xi_{\mathbf{k}}^{2}+\Delta_{s}^{2}=V^{2}$, $\mathcal{L}_{\mathbf{k}}=0$. After some straightforward calculations, we find that when $k_{x}=0,\left(\mu+2 t+2 t \cos k_{y}\right)^{2}+\Delta_{s}^{2}=V^{2}$; when $k_{x}=\pi,\left(\mu-2 t+2 t \cos k_{y}\right)^{2}+\Delta_{s}^{2}=V^{2}$. Finally, the gap closes at $\left\{k_{x}=0, \cos k_{y}=\frac{ \pm \sqrt{V^{2}-\Delta_{s}^{2}}-\mu}{2 t}-1\right\}$ or $\left\{k_{x}=\pi, \cos k_{y}=\frac{ \pm \sqrt{V^{2}-\Delta_{s}^{2}}-\mu}{2 t}+1\right\}$ subjected to $\left|\cos k_{y}\right| \leqslant 1$. Therefore, we can find that the gap closes in the regions from $\mathrm{A}$ to $\mathrm{G}$ as shown in the Fig. (1p). The number of gap-closing points at $k_{x}=0, \nu_{1}$ and $k_{x}=\pi$, $\nu_{2}$ are also shown as a pair $\left(\nu_{1}, \nu_{2}\right)$. Later we shall derive a relation between the number of gap-closing points in the first Brillouin zone and the topological invariant of the Hamiltonian. Interestingly, different from the phase diagram of the Rashba NCS in the Fig. (17), where the gap closes in some boundary lines and each gapped region between them has a distinct Chern number, the phase diagram of the Dresselhaus NCS has a gapless area from A to G as shown in the Fig. (1), which means that the system is in the semimetal phase in the whole region. Inside the gapless region, it is well known that the Chern number is not well-defined. However, several other topological invariants which are obtained from symmetry analysis of the Hamiltonian can still be used to characterize the topologically different semimetal phases in the gapless region. For the Hamiltonian Eq. (2), we enumerate several symmetries as follow: (i) particlehole symmetry, $\Xi^{-1} \mathcal{H}(\mathbf{k}) \Xi=-\mathcal{H}(-\mathbf{k})$; (ii) partial particle-hole symmetry, $\Xi^{-1} \mathcal{H}\left(k_{x}, k_{y}\right) \Xi=-\mathcal{H}\left(-k_{x}, k_{y}\right)$ and (iii) chiral symmetry, $\Sigma^{-1} \mathcal{H}(\mathbf{k}) \Sigma=-\mathcal{H}(\mathbf{k})$, where $\Xi=\sigma_{x} K, \Sigma=i \sigma_{y} \tau_{x}$ and $K$ is the complex conjugation operator. We can define the Pfaffian invariant ${ }^{29}$ for the particle-hole symmetric Hamiltonian as

$$
\mathcal{P}=\operatorname{sgn}\left\{\frac{\operatorname{Pf}\left[\mathcal{H}\left(\mathbf{K}_{\mathbf{1}}\right) \sigma_{x}\right] \operatorname{Pf}\left[\mathcal{H}\left(\mathbf{K}_{\mathbf{4}}\right) \sigma_{x}\right]}{\operatorname{Pf}\left[\mathcal{H}\left(\mathbf{K}_{\mathbf{2}}\right) \sigma_{x}\right] \operatorname{Pf}\left[\mathcal{H}\left(\mathbf{K}_{\mathbf{3}}\right) \sigma_{x}\right]}\right\},
$$

where $\mathbf{K}_{\mathbf{1}}=(0,0), \mathbf{K}_{\mathbf{2}}=(\pi, 0), \mathbf{K}_{\mathbf{3}}=(0, \pi)$ and $\mathbf{K}_{\mathbf{4}}=(\pi, \pi)$ are the four particle-hole symmetric mo- 

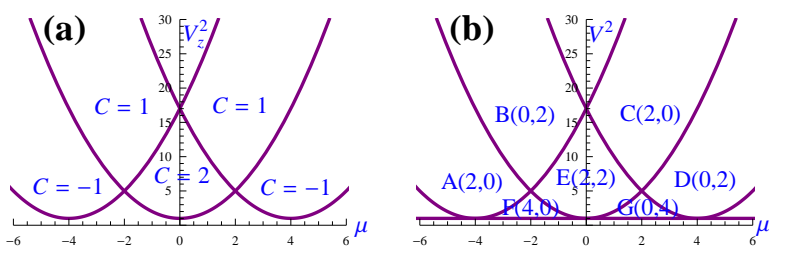

FIG. 1. (color online). The phase diagrams of $s$-wave (a) Rashba and (b) Dresselhaus NCS. The parameters are $t=1$ and $\Delta_{s}=1$. In (b), $V^{2}=V_{x}^{2}+V_{y}^{2}$. The Chern number in different regions is indicated in (a). The number of gapclosing points at $k_{x}=0, \nu_{1}$ and $k_{x}=\pi, \nu_{2}$ in different regions are also shown as a pair $\left(\nu_{1}, \nu_{2}\right)$ in (b).

menta in the first Brillouin zone of the square lattice. Similarly, the Pfaffian invariant ${ }^{30}$ for the partial particlehole symmetric system is

$$
\mathcal{P}\left(k_{y}\right)=\operatorname{sgn}\left\{\frac{\operatorname{Pf}\left[\mathcal{H}\left(\pi, k_{y}\right) \sigma_{x}\right]}{\operatorname{Pf}\left[\mathcal{H}\left(0, k_{y}\right) \sigma_{x}\right]}\right\} .
$$

For the chiral symmetry, if we take the basis where $\Sigma$ is diagonal, $\Sigma=\operatorname{diag}(i, i,-i,-i)$, then the Hamiltonian becomes off-diagonal, $\mathcal{H}(\mathbf{k})=\left(\begin{array}{cc}\mathbf{0} & q(\mathbf{k}) \\ q^{\dagger}(\mathbf{k}) & \mathbf{0}\end{array}\right)$. Using this $q(\mathbf{k})$, we can define the winding number $\frac{26}{26}$ as

$$
\mathcal{W}\left(k_{y}\right)=\frac{i}{2 \pi} \int_{-\pi}^{\pi} d k_{x} \operatorname{tr}\left[q^{-1}(\mathbf{k}) \partial_{k_{x}} q(\mathbf{k})\right] .
$$

The Pfaffian invariant $\mathcal{P}$ can be used for identifying topologically different semimetal phases of the Hamiltonian Eq. (2). It is easy to check that $\mathcal{P}_{A}=\mathcal{P}_{B}=\mathcal{P}_{C}=\mathcal{P}_{D}=$ -1 and $\mathcal{P}_{E}=\mathcal{P}_{F}=\mathcal{P}_{G}=1$ in the phase diagram of the Dresselhaus NCS as shown in the Fig. (1) . Therefore, the semimetal phases in the region of $\mathrm{A}, \mathrm{B}, \mathrm{C}, \mathrm{D}$ and the region of $\mathrm{E}, \mathrm{F}, \mathrm{G}$ are topologically inequivalent. As for the other two topological invariants $\mathcal{P}\left(k_{y}\right)$ and $\mathcal{W}\left(k_{y}\right)$, below we shall show that they can be used to determine the range of edge states in the edge Brillouin zone.

\section{MAJORANA FERMIONS AT THE EDGE OF THE SYSTEM}

To demonstrate the novel properties in the semimetal phase of the Dresselhaus NCS, we study the Andreev bound states and Majorana Fermions at the edge and in the vortex core of it. We now first turn to study the ABSs of the Dresselhaus NCS. By setting the boundary conditions of $x$ direction to be open and $y$ to be periodic, we diagonalize the Hamiltonian Eq. (2) in the cylindrical symmetry and get the edge spectra of the Hamiltonian. Interestingly, although the gap closes in the semimetal phase from region $A$ to $G$ as shown in the Fig. (1b), there exist dispersionless ABSs at the edge of the system. The two topologically different semimetal phases in the region $\mathrm{A}$ and $\mathrm{E}$ are depicted in the Fig. (2a) and (2), respectively. We would like to study the number and range of the flat ABSs in these two different semimetal phases. By the Pfaffian invariant Eq. (4) or winding number Eq. (5), the range where the flat ABSs exist in the edge Brillouin zone can be exactly obtained as shown in the Fig. (2k) and (2d). The number of flat ABSs is half of the number of gap-closing points in the first Brillouin zone. From the Hamiltonian in the chiral basis, we can see that the gap closes when $\operatorname{det} q(\mathbf{k})=0$. In the complex plane of $z(\mathbf{k})=\operatorname{det} q(\mathbf{k}) /|\operatorname{det} q(\mathbf{k})|$, a winding number can be assigned to each gap-closing point $\mathbf{k}_{0}$ as $\mathcal{W}\left(\mathbf{k}_{0}\right)=\frac{1}{2 \pi i} \oint_{\gamma} \frac{d z(\mathbf{k})}{z(\mathbf{k})-z\left(\mathbf{k}_{0}\right)}$, where $\gamma$ is a contour enclosing the gap-closing point. Due to the particle-hole symmetry, we find that $\mathcal{W}\left(\mathbf{k}_{0}\right)=-\mathcal{W}\left(-\mathbf{k}_{0}\right)$, therefore, the gap-closing points with opposite winding number are equal in number. The function $z(\mathbf{k})$ in the region $\mathrm{A}$ and $\mathrm{E}$ are shown in the Fig. (2p) and (2f). As long as the projection of opposite winding number gap-closing points does not completely overlap in the edge Brillouin zone, there will be flat ABSs connecting them 31 . Therefore, the number of flat ABSs is $\nu=\left(\nu_{1}+\nu_{2}\right) / 2$ and it is easy to check that $\mathcal{P}$ is the parity of $\nu, \mathcal{P}=(-1)^{\nu}$. The corresponding DOS of these two different semimetal phases are shown in the Fig. (2s) and (2h). We find that there is a peak at zero energy which is clearly visible in the tunneling conductance measurements. Therefore, all of these flat ABSs have clear experimental signature in the tunneling conductance measurements and the MFs predicted at the edge of the Dresselhaus NCS should be experimentally observable. As for the robustness of the flat ABSs against disorder or impurity, we can discuss it from the topological point of view. As long as the disorder or impurity does not break the symmetries of Hamiltonian Eq. (2), these flat ABSs will be protected by the three topological invariants mentioned above.

The existence of the edge states implies the nontrivial momentum space topology in the Dresselhaus NCS so that the Majorana fermions emerge at the edge of the system. In the following, we explicitly calculate the Majorana zero modes at the edge of the Dresselhaus NCS in the cylindrical symmetry. Let $x$ direction to be open and $y$ to be periodic, then by setting $k_{x} \rightarrow-i \partial_{x}$, we solve the Schrödinger equation of the Hamiltonian Eq. (2) in the real space, $H\left(k_{x} \rightarrow-i \partial_{x}, k_{y}\right) \Psi=0$, where $\Psi=\left(u_{\uparrow}, u_{\downarrow}, v_{\uparrow}, v_{\downarrow}\right)^{T}$. Due to the particle-hole symmetry in the Dresselhaus NCS, we have $u_{\uparrow}=v_{\uparrow}^{*}$ and $u_{\downarrow}=v_{\downarrow}^{*}$ at zero energy. Thus, we only need to consider the upper block of the Hamiltonian Eq. (2). For simplicity, we consider the low energy theory at $k_{x}=0$, up to the first order, we have

$$
\begin{aligned}
& \left(\varepsilon\left(k_{y}\right)-i \beta \partial_{x}\right) u_{\uparrow}+\left(V_{x}-i V_{y}\right) u_{\downarrow}+\Delta_{s} u_{\downarrow}^{*}=0, \\
& \left(\varepsilon\left(k_{y}\right)+i \beta \partial_{x}\right) u_{\downarrow}+\left(V_{x}+i V_{y}\right) u_{\uparrow}-\Delta_{s} u_{\uparrow}^{*}=0,
\end{aligned}
$$

where $\varepsilon\left(k_{y}\right)=-2 t\left(1+\cos k_{y}\right)-\mu$. Observing that $u_{\uparrow}= \pm i u_{\downarrow}^{*}$, we obtain that when $u_{\uparrow}=i u_{\downarrow}^{*}$, the solution is $u_{\uparrow}(x)=c_{1} u_{\uparrow}^{1}(x)+c_{2} u_{\uparrow}^{2}(x)$, where $c_{1}$ and 

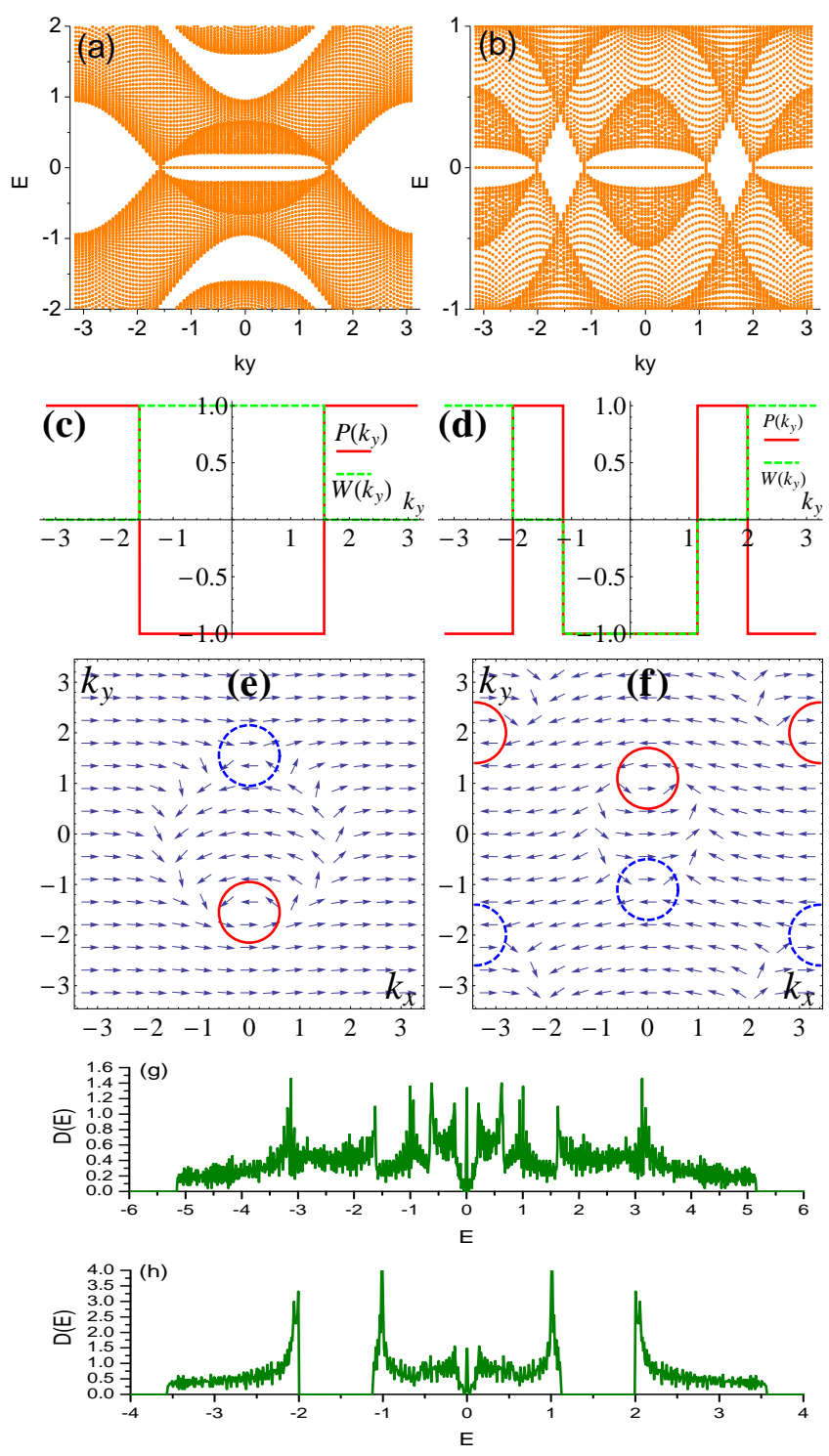

FIG. 2. (color online). (a) and (b) are the edge spectra of $s$ wave NCS with Dresselhaus SO interaction. The open edges are at $i_{x}=0$ and $i_{x}=50, k_{y}$ denotes the momentum in the $y$ direction and $k_{y} \in(-\pi, \pi]$. The parameters are $t=1, \beta=1$, $\Delta_{s}=1$ and (a) $\mu=-4, V^{2}=5$, (b) $\mu=0, V^{2}=9$, which correspond to region $\mathrm{A}$ and $\mathrm{E}$, respectively. (c) and (d) are the Pfaffian invariant Eq. (4) and winding number Eq. (5) for (a) and (b). (e) and (f) are the function $z(\mathbf{k})$ for (a) and (b). The winding number of gap-closing point enclosed by the red solid circle is 1 and by the blue dashed circle is -1 , respectively. (g) and (h) are the DOS for (a) and (b).

$c_{2}$ are real numbers and $u_{\uparrow}^{1}(x)=A_{1} e^{\lambda_{1} x}+A_{2} e^{\lambda_{2} x}$, $u_{\uparrow}^{2}(x)=i B_{1} e^{\lambda_{1} x}+i B_{2} e^{\lambda_{2} x}$, where $\lambda_{1,2}=\frac{-\Delta_{s} \mp \sqrt{V^{2}-\varepsilon^{2}}}{\beta}$ and $A_{1,2}=\frac{1}{2}\left(1 \mp \frac{V_{x}-i\left(V_{y}+\varepsilon\right)}{\sqrt{V^{2}-\varepsilon^{2}}}\right), B_{1,2}=\frac{1}{2}\left(1 \pm \frac{V_{x}-i\left(V_{y}-\varepsilon\right)}{\sqrt{V^{2}-\varepsilon^{2}}}\right)$; when $u_{\uparrow}=-i u_{\downarrow}^{*}$, the solution is similar to the case of $u_{\uparrow}=i u_{\downarrow}^{*}$. We consider the Dresselhaus NCS in the positive $x$ plane with the edge located at $x=0$. Let us assume $\Delta_{s}>0$ for simplicity, then from the solutions of the Eq. [6], the critical point for existing a normalizable wavefunction under this boundary condition is determined by $V^{2}-\varepsilon\left(k_{y}\right)^{2}=\Delta_{s}^{2}$, which is consistent with the gap-closing condition $\left(\mu+2 t+2 t \cos k_{y}\right)^{2}+\Delta_{s}^{2}=V^{2}$ at $k_{x}=0$. By the same reason, the condition for normalizable wavefunctions is consistent with the gap-closing condition $\left(\mu-2 t+2 t \cos k_{y}\right)^{2}+\Delta_{s}^{2}=V^{2}$ if we consider the low energy theory at $k_{x}=\pi$. Therefore, the Majorana bound state is $\left(u_{\uparrow}, i u_{\uparrow}^{*}, u_{\uparrow}^{*},-i u_{\uparrow}\right)^{T}$, where $u_{\uparrow}$ is the solution of Eq. (6).

\section{MAJORANA FERMIONS IN THE VORTEX CORE OF THE SYSTEM}

To further study the Majorana fermions in the Dresselhaus NCS, we consider the zero energy vortex core states by solving the BdG equation for the superconducting order parameter of a single vortex $\Delta(r, \theta)=$ $\Delta \exp (i \theta)^{32}$. To do this, the $s$-wave superconducting term in the Hamiltonian Eq. (1) is modified to be position-dependent, $H_{s}=\sum_{i}\left(\Delta e^{i \theta_{i}} c_{i \uparrow}^{\dagger} c_{i \downarrow}^{\dagger}+\right.$ H.c. $)$. We numerically solve the Schrödinger equation $H \Psi=E \Psi$ for the Hamiltonian in Eq. (1), where $\Psi=\left(u_{\uparrow}, u_{\downarrow}, v_{\uparrow}, v_{\downarrow}\right)^{T}$. At zero energy we have $u_{\uparrow}=v_{\uparrow}^{*}$ and $u_{\downarrow}=v_{\downarrow}^{*}$ as the particle-hole symmetry in the Dresselhaus NCS, then the Bogoliubov quasiparticle operator, $\gamma^{\dagger}(E)=$ $\sum_{i}\left(u_{i \uparrow} c_{i \uparrow}^{\dagger}+u_{i \downarrow} c_{i \downarrow}^{\dagger}+v_{i \uparrow} c_{i \uparrow}+v_{i \downarrow} c_{i \downarrow}\right)$ becomes Majorana operator $\gamma^{\dagger}(0)=\gamma(0)$. Therefore, below we only consider the zero energy vortex core states for discussing the MFs in the vortex core. Let's set the $x$ and $y$ directions to be open boundary, then we solve the BdG equations numerically and calculate the density profile of quasiparticle for the zero energy vortex core states. Previously, we have shown in the Fig. (2) that there is a novel semimetal phase in the Dresselhaus NCS where the zero energy flat ABSs host MFs. Here we shall ascertain whether there exist zero energy vortex core states hosting MFs in this semimetal phase. The density profiles of quasiparticle of the zero energy vortex core states are shown in the Fig. (3a) and (3b), which correspond to the region A and $\mathrm{E}$ in the phase diagram of the Fig. (1b), respectively. The numerical results of the energy for the zero energy vortex core states are $E=2.54 \times 10^{-3}$ for Fig. $(3 \mathrm{a})$ and $E=6.68 \times 10^{-3}$ for Fig. (3b), respectively. For the choice of parameters in our simulations, the order of magnitude of $\Delta_{s}^{2} / E_{F}$ is $10^{-1}$. Thus, the numerical results have much smaller energy than the Caroli-de Gennes-Matricon (CdGM) mode ${ }^{33}$. It is clear to see that there are zero energy states in the vortex core from the Fig. (3), therefore, the MFs exist in the vortex core of the Dresselhaus NCS. 

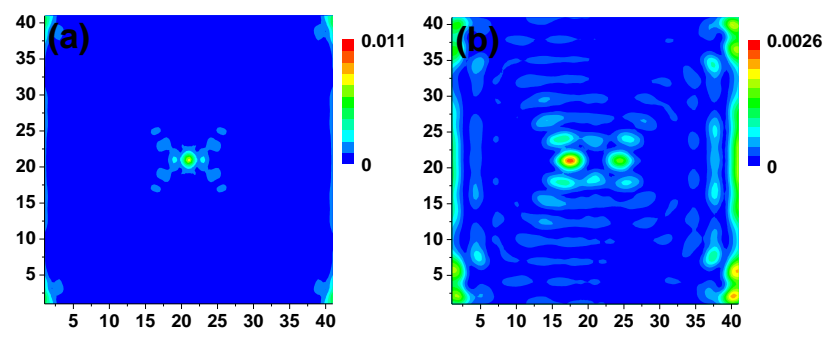

FIG. 3. (color online). The probability distribution of quasiparticle for the Dresselhaus NCS plotted on the $41 \times 41$ square lattice. The parameters are $t=1, \beta=1, \Delta_{s}=1$. The chemical potential and in-plane magnetic field are (a) $\mu=-4, V^{2}=5$ and (b) $\mu=0, V^{2}=9$.

\section{SUMMARY}

In summary, we have investigated the topological phase and Majorana fermion in the $s$-wave Dresselhaus (110) SO coupled NCS. We find that there is a gapless region appearing in the phase diagram of the Dresselhaus NCS. We observe that there exist flat Andreev bound states which host Majorana fermions in the gapless region. The chemical compound InSb has the largest Dresselhaus (110) SO coupling $\frac{[18 \mid 34}{13}$ which makes it promising to observe the MFs at the edge or in the vortex core of the system. We can fabricate an InSb (110) quantum well, in contact with an $s$-wave superconducting aluminum and couple it to an in-plane magnetic field. Thus we can apply the tunneling conductance measurements to detect the zero-bias conductance peak of the system.

\section{ACKNOWLEDGMENTS}

This work is partly supported by National Research Foundation and Ministry of Education, Singapore (Grant No. WBS: R-710-000-008-271)
* jiabinyou@gmail.com

† phyohch@nus.edu.sg

¥ phyvv@nus.edu.sg

1 G. E. Volovik, Exotic Properties of Superfluid ${ }^{3} \mathrm{He}$ (World Scientific, 1992).

2 D. J. Thouless, M. Kohmoto, M. P. Nightingale, and M. den Nijs, Phys. Rev. Lett. 49, 405 (1982).

3 F. D. M. Haldane, Phys. Rev. Lett. 61, 2015 (1988)

4 B. A. Bernevig and S.-C. Zhang, Phys. Rev. Lett. 96, $106802(2006)$

M. König, H. Buhmann, L. W. Molenkamp, T. Hughes, C.X. Liu, X.-L. Qi, and S.-C. Zhang, Journal of the Physical Society of Japan 77, 031007 (2008)

${ }^{\circ}$ C. L. Kane and E. J. Mele, Phys. Rev. Lett. 95, 226801 (2005)

C. L. Kane and E. J. Mele, Phys. Rev. Lett. 95, 146802 (2005)

${ }^{8}$ L. Fu, C. L. Kane, and E. J. Mele, Phys. Rev. Lett. 98, 106803 (2007)

${ }^{9}$ N. Read and D. Green, Phys. Rev. B 61, 10267 (2000)

10 M. Sato, Y. Takahashi, and S. Fujimoto, Phys. Rev. B 82, $134521(2010)$

11 J. Martin, S. Ilani, B. Verdene, J. Smet, V. Umansky, D. Mahalu, D. Schuh, G. Abstreiter, and A. Yacoby, Science 305, 980 (2004).

12 L. Fu and C. L. Kane, Phys. Rev. Lett. 100, 096407 (2008)

13 Y. Oreg, G. Refael, and F. von Oppen, Phys. Rev. Lett. 105, $177002(2010)$

14 A. Kitaev, Annals of Physics 303, 2 (2003)

15 T. H. Hsieh and L. Fu, Phys. Rev. Lett. 108, 107005 (2012)

${ }^{16}$ A. Kitaev, Phys. Usp. 44, 131 (2001).

17 J. Alicea, Y. Oreg, G. Refael, F. von Oppen, and M. P. A. Fisher, Nat. Phys. 7, 412 (2011).
18 J. Alicea, Phys. Rev. B 81, 125318 (2010)

19 M. Lababidi and E. Zhao, Phys. Rev. B 86, 161108 (2012)

20 V. Mourik, K. Zuo, S. M. Frolov, S. R. Plissard, E. P. A. M. Bakkers, and L. P. Kouwenhoven, Science 336, 1003 (2012).

21 S. Sasaki, M. Kriener, K. Segawa, K. Yada, Y. Tanaka, M. Sato, and Y. Ando, Phys. Rev. Lett. 107, 217001 (2011)

${ }^{22}$ J. R. Williams, A. J. Bestwick, P. Gallagher, S. S. Hong, Y. Cui, A. S. Bleich, J. G. Analytis, I. R. Fisher, and D. Goldhaber-Gordon, Phys. Rev. Lett. 109, 056803 (2012)

${ }^{23}$ J. D. Sau, R. M. Lutchyn, S. Tewari, and S. Das Sarma, Phys. Rev. Lett. 104, 040502 (2010)

24 A. P. Schnyder and S. Ryu, Phys. Rev. B 84, 060504 (2011)

${ }_{25}$ P. M. R. Brydon, A. P. Schnyder, and C. Timm, Phys. Rev. B 84, 020501 (2011).

${ }_{20}$ M. Sato, Y. Tanaka, K. Yada, and T. Yokoyama, Phys. Rev. B 83, 224511 (2011).

27 C. L. M. Wong, J. Liu, K. T. Law, and P. A. Lee, arXiv:1206.5601v3.

28 Y. Tanaka, Y. Mizuno, T. Yokoyama, K. Yada, and M. Sato, Phys. Rev. Lett. 105, 097002 (2010)

29 P. Ghosh, J. D. Sau, S. Tewari, and S. Das Sarma, Phys. Rev. B 82, 184525 (2010).

so S. Tewari and J. D. Sau, Phys. Rev. Lett. 109, 150408 (2012)

${ }^{31}$ F. Wang and D.-H. Lee, Phys. Rev. B 86, 094512 (2012).

32 M. Sato and S. Fujimoto, Phys. Rev. B 79, 094504 (2009).

33 C. Caroli, P. D. Gennes, and J. Matricon, Physics Letters 9, 307 (1964)

${ }^{34}$ M.-H. Liu and C.-R. Chang, Phys. Rev. B 82, 155327 (2010) 